\title{
The Jahn-Teller and Pseudo-Jahn-Teller Effects: A Unique and Only Source of Spontaneous Symmetry Breaking in Atomic Matter
}

\author{
Isaac B. Bersuker (D)
}

check for updates

Citation: Bersuker, I.B. The Jahn-Teller and Pseudo-Jahn-Teller Effects: A Unique and Only Source of Spontaneous Symmetry Breaking in Atomic Matter. Symmetry 2021, 13, 1577. https://doi.org/10.3390/ sym13091577

Academic Editor: Enrico Bodo

Received: 26 July 2021

Accepted: 25 August 2021

Published: 27 August 2021

Publisher's Note: MDPI stays neutral with regard to jurisdictional claims in published maps and institutional affiliations.

Copyright: (C) 2021 by the author Licensee MDPI, Basel, Switzerland. This article is an open access article distributed under the terms and conditions of the Creative Commons Attribution (CC BY) license (https:// creativecommons.org/licenses/by/ $4.0 /)$.
Department of Chemistry, The University of Texas at Austin, Austin, TX 78712, USA; bersuker@cm.utexas.edu

\begin{abstract}
In a mostly review paper, we show that the important problem of symmetry, broken symmetry, and spontaneous broken symmetry of polyatomic systems is directly related to the JahnTeller (JT) and pseudo-Jahn-Teller (PJT) effects, including the hidden-JT and hidden-PJT effects, and these JT effects (JTEs) are the only source of spontaneous symmetry breaking in matter. They are directly related to the violation of the adiabatic approximation by the vibronic and other nonadiabatic couplings (jointly termed nonadiabaticity) in the interaction between the electrons and nuclei, which becomes significant in the presence of two or more degenerate or pseudodegenerate electronic states. In a generalization of this understanding of symmetry, we suggest an improved (quantum) definition of stereo-chemical polyatomic space configuration, in which, starting with their highsymmetry configuration, we separate all atomic systems into three distinguishable groups: (1) weak nonadiabaticity, stable high-symmetry configurations; (2) moderate-to-strong nonadiabaticity, unstable high-symmetry configurations, JTEs, spontaneous symmetry breaking (SSB); (3) very strong nonadiabaticity, stable distorted configurations. The JTEs, inherent to the second group of systems, produce a rich variety of novel properties, based on their multiminimum adiabatic potential energy surface (APES), leading to a short lifetime in the distorted configuration. We show the role of the Curie principle in the possibilities to observe the SSB in atomic matter, and mention briefly the revealed recently gamma of novel properties of matter in its interaction with external perturbation that occur due to the SSB, including ferroelectricity and orientational polarization, leading to enhanced permittivity and flexoelectricity.
\end{abstract}

Keywords: spontaneous symmetry breaking; Jahn-Teller effects; Hidden Jahn-Teller effects; polyatomic space configuration

\section{Introduction}

Symmetry is one of the most widespread characteristics of systems and processes in the material world and humane relations, and it plays an outstanding role in science, engineering, aesthetics, arts, and everyday life [1-3]. In science, the motion of the units of atomic matter (atoms, molecules, solids, elementary particles) are described by equations that obey some well-known symmetries, meaning they remain invariant with respect to a variety of transformations (e.g., rotations, reflections, translations, permutations, time reversal, and Lorenz and gauge transformations) [1,2]. In general, we can state that symmetry is invariance under transformation. Actually, all the statements of symmetry invariances in atomic matter are grounded in the global assumption that space is homogeneous and isotropic, and time is uniform.

The role of symmetry invariance in science is exceptional. Not only are we able to rationalize the origin of observable properties based on symmetry principles, but we can also predict new laws and new observable properties based on the requirement of symmetry invariance. As stated by Albert Einstein in his famous paper on special relativity [4], "It is now natural for us to derive the laws of nature and to test their validity by means of the laws of invariance, rather than to derive the laws of invariance from what we believe to be the laws of nature." 
In spite of the symmetry invariances being controlled by the mentioned isotropic space and uniform time, the majority of macro and micro objects in the Universe, and the Universe itself (the "Vacuum" [5,6]) do not obey the highest possible symmetry: they are in a state of broken symmetry. In the majority of cases, the broken symmetry of an object (system) is induced by external influence. However, in many cases the symmetry is broken by internal forces, it takes place spontaneously, without external influence. Spontaneous symmetry breaking (SSB) in atomic matter implies that a high-symmetry configuration of the systems is plausible (may be expected), but it shows a configuration with lower symmetry in equilibrium, the symmetry breaking taking place under internal forces only.

"SSB in matter are very important, as they are present (explicitly or implicitly) in the majority of processes, including a variety of physical, chemical, and biological phenomena based on molecular configuration instability, and all kinds of phase transitions [7-9], as well as in the very existence of some elementary massive particles [5,6]. With regard to the properties of atomic systems, SSB plays a fundamental role. For instance, some free triatomic molecules (e.g., of the type ABA) in their equilibrium configuration are linear (i.e., with highest possible symmetry), whereas others similar are bent (i.e., with broken symmetry); in four-cycled compounds $\mathrm{C}_{4} \mathrm{H}_{4}$ is planar rectangular, $\mathrm{Si}_{4} \mathrm{H}_{4}$ is rectangular puckered, while $\mathrm{C}_{4} \mathrm{~F}_{4}$ is cis-trans puckered, and some of the $\mathrm{Si}_{4} \mathrm{~A}_{4}$ systems are rhombic puckered. Similarly, six-ring benzene-like systems may be planar, nonplanar, buckled, puckered, and so on, while transition metal coordination compounds in equilibrium configuration acquire a variety of symmetries. In these cases, the observable geometries are away from the possible high-symmetry configurations, and they can be classified as resulting from structural SSB" [10].

What is the origin of SSB in polyatomic systems? Are there general laws controlling all SSB in atomic matter? SSB takes place also in elementary particle transformations, as well as in transitions from elementary particles to nuclei, to atoms, to molecules and solids, and under phase transitions in solids. Are all these SSB independent or interdependent by some general property related to matter? With regard to atomic matter, in simple cases (e.g., triatomic molecules) the SSB can be attributed to quantized low-symmetry electronic distributions and hybridizations, but this simplified picture does not work in more complicated situations. It comes out that there is a more general law of Nature that controls all the SSB: degeneracy and pseudodegeneracy [10] (local quantized orbitals and hybridizations demonstrate the local forces that may combine to produce the degeneracies that drive the SSB). In other words, it looks like "Nature tries to avoid polyatomic equilibrium space configurations that produce two or more close-in-energy states". In this review paper, we disclose the basic properties of matter that lead to the SSB in atomic systems and show that they are all due to the Jahn-Teller effect and its extensions (a part of this idea and some of its consequences were elucidated partially in an earlier publication [10]).

\section{Origin of Spontaneous Symmetry Breaking in Atomic Matter: The Jahn-Teller Effect (JTE), Pseudo-JTE (PJTE), hidden-JTE, and hidden-PJTE}

The fundamental property of a polyatomic system to undergo spontaneous symmetry breaking induced by degeneracy, presently known as the Jahn-Teller effect (JTE), was first formulated by L. Landau in 1934 as follows: the configuration of any nonlinear polyatomic system in a degenerate electronic state undergoes spontaneous distortions that remove the degeneracy [11]. Since then, many extensions of this groundbreaking idea were revealed and developed. "The first, obvious extension of the original idea was achieved when it was shown that the spontaneous distortion may also take place when the degenerate energy level is slightly split (pseudodegenerate) [12]. This, at first sight not very significant enlargement of the JTE subjects, later on, served as a trigger of a much more important conclusion that sufficiently strong vibronic coupling between any two electronic states (often ground and excited) with any energy gap between them may lead to instability and distortion of the polyatomic configuration. This is the pseudo JTE (PJTE) (see in refs. [7-9,13]). More recently, it was also shown that there may be "hidden" JTE and "hidden" PJTE when the state under consideration is neither degenerate, nor pseudodegenerate, but there is 


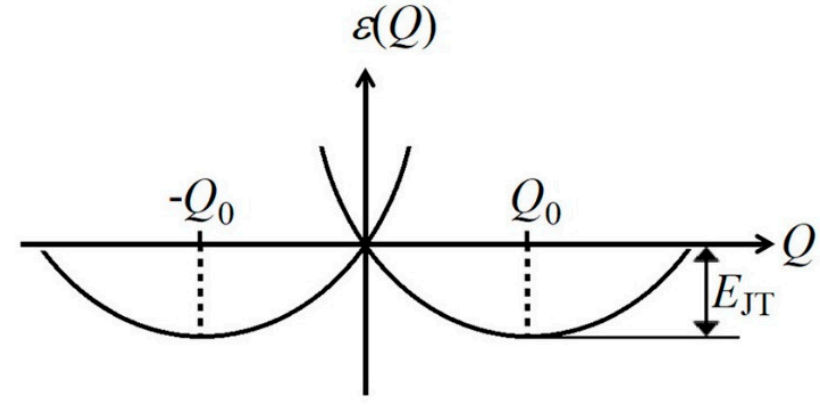

a

a strong JTE or PJTE in the excited state that penetrates the ground state [14,15]. With the PJTE and hidden JTE and PJTE added to the original JTE, we conclude that there are no polyatomic systems that can a priori be excluded from the possibility of spontaneous symmetry breaking (SSB). Moreover, it was proved that the JTE and the PJTE are the only source of instability and spontaneous distortions of high-symmetry configurations of polyatomic systems (see refs. $[7,13,16,17]$ ). Actually, degeneracy and pseudodegeneracy are the only sources of SSB in the whole spectrum of transformations in matter from elementary particles to nuclei, to atoms, molecules, and solids" [10]. Figures 1-3 show some simple illustrative features of the JTE, PJTE, hidden-JTE, and hidden-PJTE (for more information see [7-11,13,14]). Below we label this group of four JT effects as JTEs.

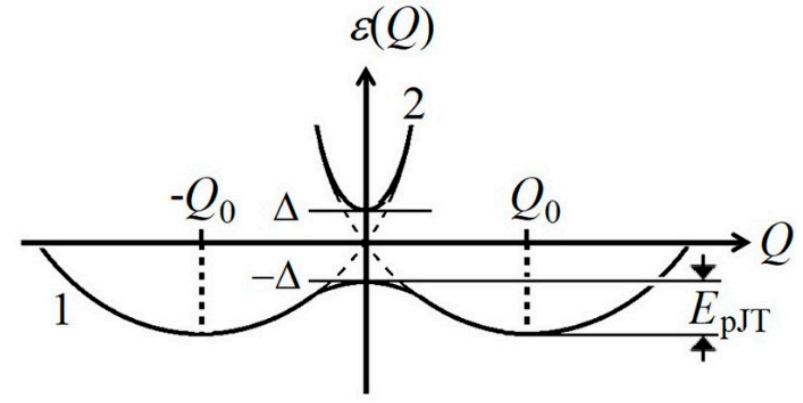

$b$

Figure 1. Some illustrative features that define the JTE and PJTE: (a) there is no minimum of the adiabatic potential energy curves at the point of two-fold degeneracy at their crossing $Q=0$ in the space of the low-symmetry nuclear displacements $Q$, meaning instability along $Q$ distortions, but the system may be stabilized in two distorted configurations at $\pm Q_{0}$ (this is the simplest picture of the JTE); (b) A similar effect takes place when the two electronic states are not degenerate, but their separation $2 \Delta$ at $\mathrm{Q}=0$ follows the condition $\Delta<\mathrm{F}^{2} / \mathrm{K}_{0}$, where $\mathrm{F}$ is the vibronic coupling constant, and $\mathrm{K}_{0}$ is the primary force constant (this is the simplest PJTE) [7].
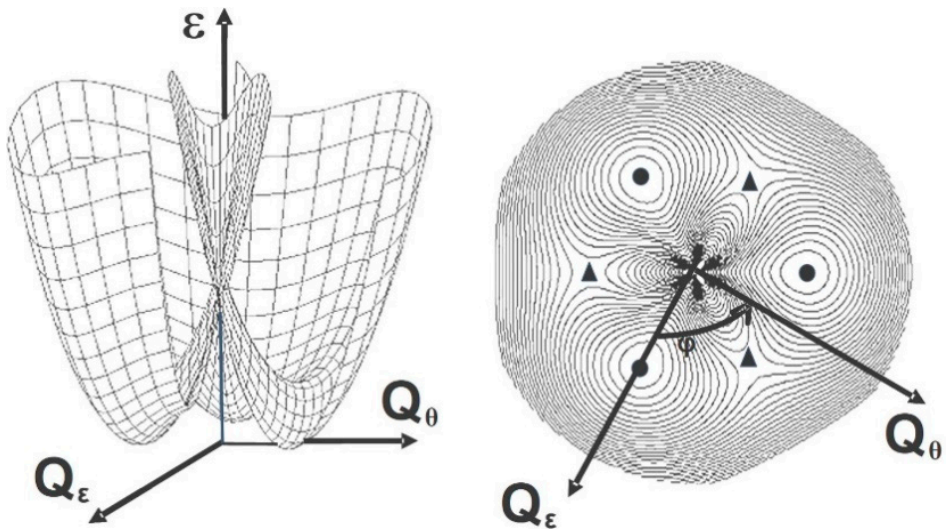

Figure 2. When the twofold degenerate electronic E state interacts with two active distortion coordinates $Q_{\vartheta}$ and $Q_{\varepsilon}$, the adiabatic potential energy surface (APES) induced by the JTE becomes more complicated with three equivalent minima points (black points in the cross-section) and three equivalent saddle points (triangles in the cross-section). This is the simplest case illustrating the multiminimum feature of the APES, which is also present in all the more complicated cases of the JTEs [7]. Reprinted from Ref. [7], copyright I. B. Bersuker. 


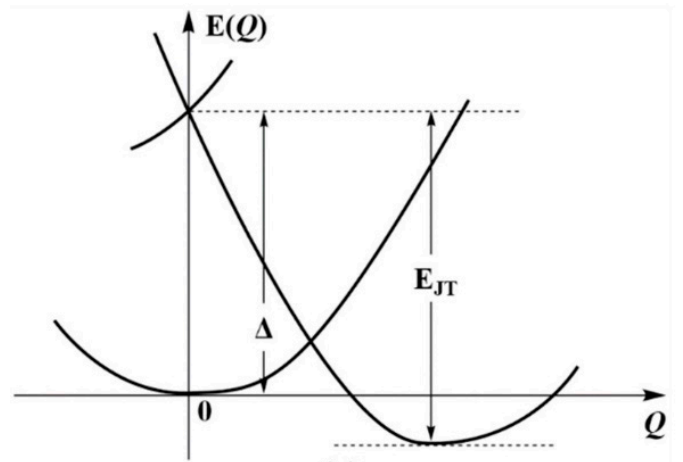

(a)

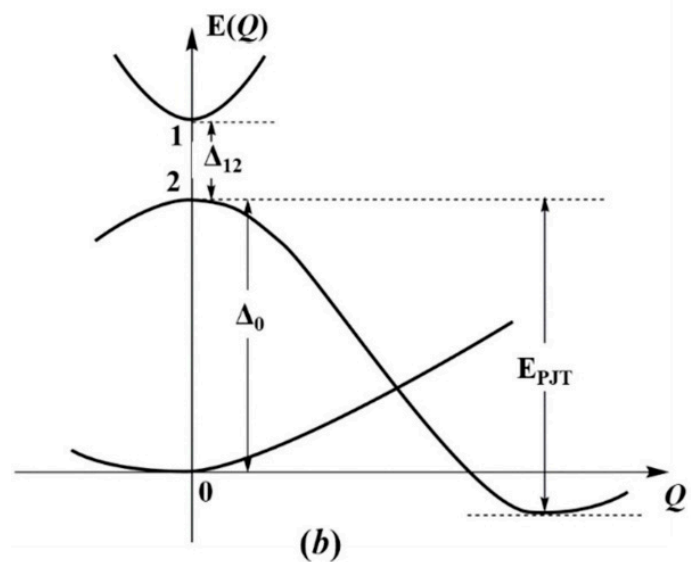

Figure 3. Illustration to the origin of the hidden-JTE and hidden-PJTE: (a) The system in a stable nondegenerate electronic state has a relatively low-lying (at the energy gap $\Delta_{0}$ ) double-degenerate excited state with a strong JTE-induced distortion, its stabilization energy $\mathrm{E}_{\mathrm{JT}}$ being larger than $\Delta_{0}$, thus producing a more stable ground state distorted along $Q$; this is the hidden-JTE; (b) In a system with a similar nondegenerate ground state as in (a), the excited state is nondegenerate, too, but there is a near-neighbor next excited state (with a relatively small energy gap between them $\Delta_{12}$ ), which allows for their PJT coupling with $\mathrm{E}_{\mathrm{PJT}}>\Delta_{0}$, resulting in a distorted ground state, similar to the case (a); this is the hidden-PJTE $[9,14,15]$.

\section{Improved (Quantum) Definition of Stereo-Chemical Space Configuration of Polyatomic Systems with Regard to Symmetry}

As illustrated above in Figures 1-3, the simplest types of the four JT effects (JTEs), starting with a high-symmetry configuration in a given electronic state, the instability and consequent SSB take place due to the presence of other active electronic states involved in the process (and this aspect of the problem is inherent to the whole variety of JTEs [7,13]). In other words, in all the cases of JTEs, there are two or more electronic states, which are coupled by the nuclear displacement, leading to SSB. This illustrates the idea that SSB in all these cases is directly related to degeneracy or pseudodegeneracy. Why does this take place and what is the role of the degeneracies?

Spontaneous symmetry breakings are induced by the JTEs that emerge from the first principles due to the non-adiabatic interactions between the electrons and nuclei [7,11-13]. Strictly speaking, these SSB, especially the multiminimum nature of their APES, require a revision of the "classical" notion of polyatomic space configuration (molecular shapes, crystal local and bulk structures, clusters, two-dimensional systems, thin films, quantum dots, qubits, etc.) and related properties. Indeed, "in quantum-mechanical description of the motions of the electrons and nuclei, the notion of space configuration is introduced in the adiabatic approximation, in which it is assumed that due to the huge difference between the masses of electrons and nuclei, the fast moving electrons follow adiabatically the much slower nuclear motions. Here adiabatic means that at each instantly fixed nuclear 
configuration, a stationary electronic charge distribution is achieved, while the nuclei move in the averaged field of the electrons. Under these conditions, the description of the motions of nuclei and electrons can be (approximately) separated. Then, by calculating the energy of the system as a function of nuclear coordinates, the APES, one finds the points of minimum energy. The nuclear coordinates at these points define the local equilibrium configuration of the system" [9].

But the adiabatic approximation is (very often) violated, thus reducing the accuracy of the classical definition of polyatomic configurations as stable geometrical formations at equilibrium. The adiabatic approximation is especially vulnerable, and may be significantly violated when for a given nuclear configuration, there are two or more close in energy, degenerate or relatively near-degenerate (pseudodegenerate) electronic states that become mixed (coupled) under nuclear displacements. Indeed, in these cases, in classical language, "the electrons have additional "degrees of freedom" that allows them to avoid (partially) the necessity to follow adiabatically the slower nuclear displacements (in first-principle calculations, the off-diagonal matrix elements of vibronic coupling and the operator of nonadiabacity that involves two or more electronic states acquires significant values [13]), thus violating the adiabatic approximation and the accuracy of the notion of nuclear configuration: there is no minimum of the APES at these points. The JTE and PJTE emerge as the corrections to the notion of polyatomic configuration, which compensate for the violation of the adiabatic approximation.

The JTEs provide for a general (rigorous) explanation of the origin of all SSB in polyatomic systems, for which the notion of space configuration is valid. With these effects included, we obtain the improved (quantum) definition of polyatomic space configuration. Under this definition "there are three types of polyatomic configurations with significant differences in related observable properties with regard to SSB. To the first type belong polyatomic systems in stable high-symmetry configurations with no electronic degeneracy or pseudodegeneracy in the ground and low-lying excited states, and hence, no explicit or hidden JTE and PJTE, meaning systems with negligible structural non-adiabaticity. For these systems, the improved definition coincides with the widespread "classical" meaning of configurations that are stable in the classical high-symmetry nuclear arrangement, except for the significant limitations in systems to which this definition applies, making them rather exceptional.

The second type of systems are those with instabilities of the high-symmetry configuration induced by the JTE and PJTE, as well as the hidden JTE and PJTE (Section 2), resulting in SSB with small to moderate distortions, the latter being of dynamic nature. Because of these effects, assumed static configurations (with small normal vibrations) become configurational-dynamic, due to two or several, or an infinite number of equivalent minima of the APES [7] (see, e.g., Figure 2). These are the systems with a rich variety of novel properties, partially demonstrated in books and reviews (see [7-13] and references therein).

The third group of systems are those with very strong non-adiabaticity in the highsymmetry configuration, for which the JTEs result in two or several equivalents, or in an unlimited number of equivalent distorted configurations (in a trough), with a very high stabilization energy by distortion. The systems under this definition, once created in the distorted configuration, remain there forever, and in this respect, they do not challenge the classical image of a stable low-symmetry configuration. For these cases, the larger definition tells one why the system is not in its possible high-symmetry configuration" [9], and more specifically, what is the origin of its deviation from the (plausible) high-symmetry configuration in terms of JTEs parameters.

Polyatomic systems of the second group with relatively easily observable SSB dynamics in the space configuration possess a gamma of special properties, known as JTEs. Compounds of the first group with stable high-symmetry configurations and the third group with stable low-symmetry configurations, at first sight, fully coincide with their widespread "classical" definitions. However, the knowledge, that their stabilities are just particular cases of polyatomic configuration with either very weak nonadiabaticity, as in 
the compounds of the first group, or very strong nonadiabaticity in the third group, is very important, as it allows for manipulations of their properties by means of external influence. Indeed, as mentioned above, the nonadiabaticity is directly reflected in the JTEs parameters (degeneracies and pseudodegeneracies, energy gaps, vibronic coupling constants, primary force constants [7]). Direct manipulation of structural properties of systems from the third group was realized by means of external influence targeting their JTEs parameters (see references in Section 4).

Note, that there are no distinct borders between the three groups of compounds, and it is not always known a priori to which group the system under consideration belongs. For example, "the ammonia molecule $\mathrm{NH}_{3}$ is unstable in the high-symmetry planar configuration because of the PJTE instability of the latter [13], but the moderate barrier between the two equivalent pyramidal structures allows for the tunneling between them, a nonclassical property, making this system pertain to the second group. However, in a very similar molecule $\mathrm{AsH}_{3}$ with formally the same, but much stronger PJTE in the planar geometry, the barrier between the distorted pyramidal configurations becomes too large with the tunneling frequency negligible, so its configuration belongs to the third group of well-defined classical low-symmetry structures. In between these two examples, there is a similar molecule, $\mathrm{PH}_{3}$, with very slow, but still observable tunneling effect, reflecting the blur border between the three groups of configurations, defined above" [9].

\section{Experimental Observation of Spontaneous Symmetry Breaking. The Curie Principle}

Experimental observation of symmetry properties of polyatomic systems with SSB induced by JT effects is not trivial and may encounter significant difficulties. Some fundamental rules in this respect were first formulated by Pierre Curie in 1894 [18]. They state:

1. "If certain causes yield the known effects, the symmetry elements of the causes should be contained in the generated effects;

2. If the observable effects manifest certain dissymmetry (absence of symmetry elements), this latter should be contained in the causes, which have generated those effects;

3. The converse to these two previous propositions is not true, at least in practical, i.e., the effects may have higher symmetry than the causes, which generate these effects" [18].

In a shorter reformulation, this means that the observable effects may occasionally have the same or a higher symmetry than the causes, but the latter cannot have a higher symmetry than the effects produced. Curie also stated that asymmetry is essential to characterize physical phenomena: "What is necessary is that certain symmetry elements are missing: Asymmetry is what creates the phenomenon" ([18], p. 400) (Curie's principle is used directly as a postulate in quantum field theory, assuming that the observable part of the Universe (the "vacuum") is in a state of broken symmetry $[5,6])$.

In application to atomic systems, Curie's principle implies that the symmetry of the observable phenomena (the effect) may not be lower than the combined symmetry of the system plus the perturbation of observation (the causes). This means that the broken symmetry in systems with SSB can be observed only by specific types of measurements.

As mentioned above in Section 2, JTEs-induced SSB in polyatomic systems result in multiminimum APES, which in entirety, "at face value", do not violate the symmetry stipulated in the originally assumed high-symmetry reference configuration. Indeed, starting with the latter, we notice that any SSB, as a subgroup of its group of symmetry, occurs in two or several or an infinite number of equivalent distorted configurations that complement each other to the original high symmetry. For example, if there is a spontaneous tetragonal distortion of a free octahedron with identical atoms in the octahedral positions, it occurs equivalently along its three tetragonal axes; its APES in the space of tetragonal distortions has three equivalent minima (Figure 2), in each of which the system has lower symmetry, than the initial (reference) one. In some of such free limit-size polyatomic systems with a multiminimum APES the ground-state wavefunction is a totally symmetric linear combination of the wavefunctions in the minima (if they are not orthogonal). 
This quantum-mechanically averaged nuclear dynamic description of the system prompted some authors to state that SSB is not possible in finite atomic systems (see, e.g., [19]). Such statements follow from unawareness about the JT effects, some of which produce local SSB in systems, in which the tunneling between the equivalently distorted configurations is practically impossible $[7,13]$. For instance, the three mutually orthogonal configurations produced by the JTE problem $T \otimes e$ in polyatomic system with a threefold degenerate $T$ term, or in the $E \otimes b_{1}$ problem in some systems with a twofold degenerate $E$ term, etc., the electronic wavefunctions in the minima are mutually orthogonal, so no tunneling between them is allowed. (It was shown recently that this restriction may be removed by external magnetic field influence [20]). In these cases, the free system of finite (molecular) size remains in the spontaneously broken symmetry permanently (at least at $\mathrm{T}=0)$.

"Even when there is a theoretical possibility of tunneling between the equivalent SSB configurations, the barrier between them and the lifetime of the system in the distorted configuration may be very large (practically infinite), so they can be quite observable. There is a vast (infinite) variety of chemical and biological systems in which the SSB is induced by a degeneracy in one of its centers involving the whole chemically bonded system. For them the tunneling between the equivalent broken-symmetry configurations is practically impossible because of their polyatomic heavy weight. Examples are plenty: see, for instance, $\mathrm{Si}_{4}$ (Emind) ${ }_{4}$ (Emind = bulky 1,1,7,7-tetraethyl-3,3,5,5-tetramethyl- s-hydrindacen4-yl), which is in broken symmetry due to the PJTE-induced planar-rhombic $S_{4}$ center [21], or hemoglobin with the Fe atom out of plane of the porphyrin ring due to the PJTE [22]. In such systems no tunneling between the equivalent distorted configurations is expected in years" [10].

The Curie's principle limitations of external perturbations that allow for observation of these SSB can be formulated here in simple (practical) terms. Denote the "lifetime" of the SSB system in one of the equivalent minima by $\tau$ and the characteristic time of effective interaction with the external perturbation, the "time of measurement", by $\tau^{\prime}$ (e.g., the period of a spectroscopic, electromagnetic or ultrasound wave). Then the SSB will be seen in the measurement if

$$
\tau^{\prime}<\tau
$$

If $\tau^{\prime}>\tau$, the distorted configuration of the system will not be seen with the given method of observation, because during the time of its characteristic interaction with the system the latter is in time to average its flexible multi-configurational SSB.

The condition (1) is a particular case of Curie's principle leading to the rule of relativity of the observed SSB to the means of observation [7], when the broken symmetry is only seen in measurements with methods for which $\tau^{\prime}<\tau$. Significantly different $\tau^{\prime}$ values are inherent in spectroscopies such as EXAFS, EPR, NMR, optical, vibrational, Mossbauer, and ultrasound, where $\tau^{\prime}$ characterizes their wavelengths. Examples of this relativity to the means of observation of SSB are vast [7-10,13]. We can imagine a multiminimum system with the mentioned above very large (infinite) energy barriers between the equivalent minima, for which $\tau$ is very large (infinite), meaning the states in the equivalent minima are practically orthogonal, and hence the SSB is observable with any method. These are atomic systems in equilibrium with very strong SSB induced by nonadiabacity, outlined in Section 3 as polyatomic systems from the third group.

As mentioned in Section 3, the knowledge that the distorted configurations of polyatomic systems are due to (and only to) the JTE or PJTE, is important by itself, even when they are from the third group of systems in the above classification with very large $\tau$ values. Indeed, this knowledge allows manipulating the structure and properties of such systems by means of external perturbations, targeting the JTE or PJTE parameters [23-25]. Such manipulations were realized in a series of two-dimensional systems with puckered or buckled out-of-plane distortions induced by the PJTE, by restoring their planar configuration with external influence $[23,24]$, and in some hexaferrite crystals, by creating a sublattice of JTE centers via changing their oxidation state with redox dopants [25]. 
If $\tau$ is small and $\tau<\tau^{\prime}$, the broken symmetry configuration is not seen directly with the given method of observation. This may be termed as "hidden broken symmetry" (in Curie's principle formulation, the symmetry in the effect cannot be lower than in the cause, including the symmetry elements of the system plus perturbation of measurement). Note, however, that the observable properties of such multiminimum systems with highfrequency (small $\tau$ ) dynamic SSB, which exhibit an averaged high-symmetry configuration in some experiments, are still significantly different from that of a stable high-symmetry configuration without SSB. These cases became most important recently, in view of the revealed novel applications, outlined in the next section.

\section{Cooperative Interaction of Systems with SSB and Interactions with External Perturbations}

The properties of systems with multiminimum APES induced by the JT effects are essentially different from those of stable single-minima ones. First, we refer to the traditional local distortions, induced by the JT effects, which may be observed directly under certain external perturbations with the above limitation $\tau^{\prime}<\tau$. The latter can be overcome in a variety of experimental methods, involving external electric and magnetic fields, and especially in all kinds of spectroscopies. Relatively strong SSB distortions in the local centers in crystals may lead to their cooperative interactions and structural phase transitions. Publications on such local and cooperative SSB in polyatomic systems are enumerable, they appear in the literature under the names of the JTE and PJTE (see, e.g., the books and latest reviews $[7-11,13,14]$ and references therein). The hidden-JTE and hidden-PJTE are relatively novel [14], and hence not widely involved so far (however, see [15,26]).

In the limit case of small lifetime $\tau$ of the system in the minima of the multiminimum APES, the direct observation of the distortions becomes difficult. For this reason, the cases of small SSB in polyatomic systems were ignored for a long time. However, in more recent works, it was shown that the SSB in systems with relatively small lifetimes $\tau$ in the minima wells of the multiminimum APES are of special interest, they became most challenging in view of the recently revealed novel properties in their cooperative interactions and interactions with external perturbations (see [27-35] and references therein).

First, we notice that the small lifetime $\tau$ value in systems with SSB indicates that there is relatively high-frequency (low-barrier) tunneling between its equivalent minima of the APES. This in turn means that such systems are very sensitive to the influence of the environment. Indeed, because of the small energy barriers between the equivalently distorted configurations in the minima of the APES, any small external perturbation of lower symmetry stabilizing the system in one of them (oriented along the external perturbation), thus violating the conditions of tunneling between the equivalent minima. This results in the observable broken-symmetry configurations, expected due to SSB (in the literature often called static JTE). Because of the multiminimum nature of the APES, different low-symmetry influences of the environment may lead to differently distorted stable structures. In other words, the same or similar systems with SSB may acquire different distorted configurations under a relatively very weak external influence (e.g., next coordination sphere in crystals). It follows that with regard to the influence of the environment polyatomic systems with SSB, induced by JT effects, possess some special properties of plasticity [27]. This JT-induced plasticity leads to the formation of a variety of specific crystal structures with the same or similar origin. It was demonstrated on a class of coordination compounds of $\mathrm{Cu}$ (II) [27].

Most interesting properties were revealed in polyatomic systems with SSB when the distortion in the minima of the APES is of dipolar nature [28-33]. It was shown that such dipolar distortions may occur in centrosymmetric systems under the PJTE, and they lead to a variety of properties of crystals with such centers (e.g., $\mathrm{BaTiO}_{3}$ ), confirmed experimentally $[28,29]$. The PJTE was shown to be the unique origin of the whole series of properties of such perovskite crystals, including ferroelectricity, multiferroicity, flexoelectricity, permittivity, and polar nanoregions [28-35]. 
All these effects emerge from the dynamics of the SSB induced by the JT effects, which is very sensitive to external influence. The study of such SSB-induced novel properties is far from being exhausted. In particular, quite recently, a novel effect is predicted, based on these studies: orientational polarization of solids [32-35]. The tunneling between the differently oriented dipolar distortions in the equivalent minima of the SSB makes the crystals with such centers similar to polar liquids (the possible existence of crystals with free rotating dipoles above the freezing temperatures was predicted by Debye in 1912, but not found until the present works [32-35]). Under the influence of external electric fields, the equivalent minima of the APES become nonequivalent with all the dipole displacements frozen in the minima along the electric field, meaning orientational polarization, which results in strongly enhanced permittivity and electrostriction [33,34]. A similar effect takes place under a local strain gradient leading to enhanced flexoelectricity [32]. The orientational polarization is confirmed experimentally as being by orders of magnitude stronger, than the displacive polarization, expected in the absence of JTE-induced SSB [32-35].

\section{Conclusions}

The main goal of this brief presentation of the symmetry problem in the structure and properties of polyatomic systems, in context with the spontaneous symmetry breaking induced by the series of Jahn-Teller effects, is to promote a better understanding of their background property, the polyatomic space configuration and its role in the related phenomena. We refer first to the four forms of the JT effects, JTE, PJTE, and their two hindered forms, $h$-JTE and $h$-PJTE (jointly JTEs), which together exhaust all possible causes of spontaneous symmetry breaking of polyatomic systems when starting with their highsymmetry configuration. In all these four cases, there are two or more electronic states that become coupled under the nuclear displacements, triggering nonadiabatic effects. Then, we show that with regard to the latter, polyatomic systems, starting with their high-symmetry configuration, can be separated, approximately, into three groups: weak to zero non-adiabaticity (stable high-symmetry configurations), relatively moderate to strong nonadiabaticity (instability and JTEs leading to SSB with multiminimum APES), and very strong nonadiabaticity (stable, low-symmetry configurations), and there are no well-defined borders between the three groups.

Most interesting is the second group of systems, which demonstrate SSB with a rich variety of specific properties induced by the JTEs via their multiminimum APES. In addition to the already traditional demonstrations of these effects in physical, chemical, and biological phenomena, as well as in materials science, we mention here the newly developed methods of manipulation of the structure and properties of such systems by means of external influence, targeting the JTE parameters, and the revealed recently series of novel properties of systems with polar distortions in their interaction with external perturbation. Among these findings, we refer to the revealed earlier plasticity effect, and to the newly discovered orientational polarization of solids that yields giant permittivity, flexoelectricity, and electrostriction.

Funding: This research received no external funding.

Institutional Review Board Statement: Not applicable.

Informed Consent Statement: Not applicable.

Data Availability Statement: Not applicable.

Conflicts of Interest: The author declares no conflict of interest.

\section{References}

1. Weyl, H. Symmetry; Princeton University Press: Princeton, NJ, USA, 1952.

2. Houtappel, R.M.F.; van Dam, H.; Wigner, E.P. The conceptual basis and use of the geometric invariance principles. Rev. Mod. Phys. 1965, 37, 595-632. [CrossRef]

3. Hargittai, I. Symmetry: Unifying Human Understanding; Pergamon Press: Oxford, UK, 1989. 
4. On the electrodynamics of moving bodies. In The Principle of Relativity; Einstein, A.; Lorentz, H.A.; Minkowski, H.; Weyl, H. (Eds.) Dover Books: New York, NY, USA, 1952; pp. 35-65.

5. Weinberg, S. Quantum Theory of Fields; Cambridge University Press: Cambridge, UK, 1995.

6. Nagashima, Y. Elementary particle physics. In Foundation and the Standard Model; John Wiley and Sons, Inc.: New York, NY, USA, 2010; Volume 2.

7. Bersuker, I.B. The Jahn-Teller Effect; Cambridge University Press: Cambridge, UK, 2006.

8. Bersuker, I.B. The pseudo Jahn-Teller effect-A two-state paradigm in formation, deformation, and transformation of molecular systems and solids. Chem. Rev. 2013, 113, 1351-1390. [CrossRef]

9. Bersuker, I.B. Jahn-Teller and Pseudo-Jahn-Teller Effects: From particular features to general tools in exploring molecular and solid state properties. Chem. Rev. 2021, 121, 1463-1512. [CrossRef]

10. Bersuker, I.B. Spontaneous symmetry breaking in matter induced by degeneracy and pseudodegeneracy. In Advances in Chemical Physics; Rice, S., Dinner, R., Eds.; Wiley: New York, NY, USA, 2016; Volume 160, pp. 159-220.

11. Teller, E.; Englman, R. The Jahn-Teller Effect in Molecules and Crystals; Wiley: New York, NY, USA, 1972.

12. Opik, U.; Pryce, M.H.L. Studies of the Jahn-Teller effect. I. A survey of the static problem. Proc. R. Soc. London A 1957, $238,425$.

13. Bersuker, I.B.; Polinger, V.Z. Vibronic Interactions in Molecules and Crystals; Springer: New York, NY, USA, 1989.

14. Bersuker, I.B. Recent developments in the Jahn-Teller effect theory. In The Jahn-Teller Effect. Fundamentals and Implications for Physics and Chemistry; Koppel, H., Yarkony, D.R., Barentzen, H., Eds.; Springer Series of Chemical Physics; Springer: Berlin/Heidelberg, Germany, 2009; Volume 97, pp. 3-23.

15. Bersuker, I.B. Hidden Jahn-Teller and Pseudo-Jahn-Teller effects, Encyclopedia, 2020, General \& Theoretical Physics, Topic Review. Available online: https:/ / encyclopedia.pub/4283 (accessed on 20 June 2021).

16. Bersuker, I.B.; Gorinchoy, N.N.; Polinger, V.Z. On the origin of dynamic instability of molecular systems. Theor. Chim. Acta 1984, 66, 161-172. [CrossRef]

17. Bersuker, I.B.; Balabanov, N.B.; Pekker, D.M.; Boggs, J.E. Pseudo Jahn-Teller origin of instability of molecular high-symmetry configurations: Novel numerical method and results. J. Chem. Phys. 2002, 117, 10478-10486. [CrossRef]

18. Curie, P. Sur la Symmetrie dans les phenomene physiques: Symmetrie d'un champ electriques et d'un champ magnetique. J. Phys. 1894, 3, 393-415.

19. Brading, K.; Castellani, E. Symmetry and symmetry breaking. In The Stanford Encyclopedia of Philosophy, Spring 2013 ed.; Zalta, E.N., Ed.; Stanford Encyclopedia of Philosophy: Stanford, CA, USA, 2013; Available online: http:/ / plato.stanford.edu/archives/ spr2013/entries/symmetry-breaking/ (accessed on 29 September 2015).

20. Averkiev, N.S.; Bersuker, I.B.; Gudkov, V.V.; Zhevstovskikh, I.V.; Baryshnikov, K.A.; Sarychev, M.N.; Zherlitsyn, S.; Yasin, S.; Korostelin, Y.V. Magnetic field induced tunneling and relaxation between orthogonal configurations in solids and molecular systems. Phys. Rev. B 2017, 96, 094431. [CrossRef]

21. Liu, Y.; Wang, Y.; Bersuker, I.B. Geometry, electronic structure, and pseudo Jahn-Teller Effect in tetrasilacyclobutadiene analogues. Sci. Rep. 2016, 6, 23315. [CrossRef] [PubMed]

22. Bersuker, I.B.; Stavrov, S.S. Structure and properties of metalloporphyrins and hemoproteins: The vibronic approach. Coord. Chem. Rev. 1988, 88, 1-68. [CrossRef]

23. Bersuker, I.B. Manipulation of structure and properties of two-dimensional system employing the pseudo Jahn-Teller effect. FlatChem 2017, 6, 11-27. [CrossRef]

24. Gorinchoy, N.N.; Bersuker, I.B. Origin of Puckering (Buckling) of planar heterocycles and methods of its suppression. In Heterocycles: Synthesis, Reactions and Applications; Danielsen, J.M., Ed.; Nova Science Publishers: Long Island, NY, USA, $2020 ;$ pp. $129-188$.

25. Gudkov, V.V.; Sarychev, M.N.; Zherlitsyn, S.; Zhevstovskikh, I.; Averkiev, N.S.; Vinnik, D.; Gudkova, S.; Niewa, R.; Dressel, M.; Alyabyeva, L.N.; et al. Sub-lattice of Jahn-Teller centers in hexaferrite crystal. Sci. Rep. 2020, 10, 7076-7091. [CrossRef]

26. Bersuker, I.B. Spin crossover and magnetic-dielectric bistability induced by hidden pseudo-Jahn-Teller effect. Magnetochemistry 2020, 6, 64. [CrossRef]

27. Gazo, J.; Bersuker, I.B.; Garaj, J.; Kabesova, M.; Kohout, J.; Langfelderova, H.; Melnik, M.; Serator, M.; Valach, F. Plasticity of the coordination sphere of Cooper(II) complexes, its manifestations and causes. Coord. Chem. Rev. 1976, 19, 253-297. [CrossRef]

28. Bersuker, I.B. On the origin of ferroelectricity in perovskite-type crystals. Phys. Lett. 1966, 20, 589. [CrossRef]

29. Bersuker, I.B. The vibronic (Pseudo Jahn-Teller) theory of ferroelectricity. Novel aspects and applications. Ferroelectrics 2018, 536, 1-59. [CrossRef]

30. Bersuker, I.B. Pseudo Jahn-Teller origin of perovskite multiferroics, magnetic-ferroelectric crossover, and magnetoelectric effects. The d0-d10 problem. Phys. Rev. Lett. 2012, 108, 137202. [CrossRef]

31. Polinger, V.Z. Ferroelectric phase transitions in cubic perovskites. J. Phys. Conf. Ser. 2013, 428, 012026. [CrossRef]

32. Bersuker, I.B. Pseudo Jahn-Teller effect in the origin of enhanced flexoelectricity. Appl. Phys. Lett. 2015, 106, 022903. [CrossRef]

33. Bersuker, I.B. Giant permittivity and electrostriction induced by dynamic Jahn-Teller and pseudo Jahn-Teller effects. Appl. Phys. Lett. 2015, 107, 202904. [CrossRef]

34. Polinger, V.; Bersuker, I.B. Pseudo Jahn-Teller effect in permittivity of ferroelectric perovskites. J. Phys. Conf. Ser. 2017, 833, 012012. [CrossRef]

35. Bersuker, I.B.; Polinger, V. Perovskite Crystals: Unique Pseudo-Jahn-Teller origin of ferroelectricity, multiferroicity, permittivity, flexoelectricity, and Polar Nanoregions. Condens. Matter 2020, 5, 68. [CrossRef] 